\title{
Analysis and application of Bacillus subtilis sortases to anchor recombinant proteins on the cell wall
}

\author{
Hoang Duc Nguyen ${ }^{1,2,3^{*}}$, Trang Thi Phuong Phan ${ }^{1,2,3}$ and Wolfgang Schumann ${ }^{1}$
}

\begin{abstract}
Bacillus subtilis codes for two putative sortases, YhcS and YwpE, and two surface proteins, YhcR and YfkN, harboring sorting motifs supposed to be recognized by the putative sortase(s). However, there is no experimental evidence to show a direct link between these sortases and sorting sequences. To study the role of these two putative sortases on displaying YhcR and YfkN on the cell wall, expression of yhcS and ywpE was analyzed by transcriptional fusions and by Northern blot. It turned out that yhcS gene is expressed at a higher level during the late stationary phase from both experiments, while ywpE expression is not confirmed in the Northern blot analysis. Next, we constructed yhcS and ywpE single and double knockout strains and plasmids that express one or both genes to restore the functions of the knockout strains. It could be shown that display of YhcR and YfkN on the surface depended on the presence of YhcS while YwpE seems not to play a major role if any as a sortase. Finally, the putative sorting motif together with a 123-amino-acid spacer derived from YhcR and YfkN designated YhcR123 and YfkN123, respectively, were fused to an $\alpha$-amylase reporter enzyme. The fusion protein YhcR123-AmyQ could be displayed on the surface at high amounts, while YfkN123-AmyQ could be hardly detected. We conclude that the sortase YhcS can recognize and anchor YhcR on the cell wall. This result further indicates that the YhcR sorting sequence can be used to display recombinant proteins on the surface of $B$. subtilis cells.
\end{abstract}

Keywords: Sortase, B. subtilis, YhcR, YhcS, surface display, microbiorobot

\section{Introduction}

Cell surface display of recombinant proteins is usually achieved through a translational fusion of the target protein to one of the naturally occurring surface proteins of the host cell. Display of proteins on the surface of microorganisms, enabled by means of recombinant DNA technology, has become an increasingly used strategy in various applications in microbiology, biotechnology and vaccination (Samuelson et al. 2002; Wernerus and Stahl 2004; Daugherty 2007).

From a practical point of view, Gram-positive bacteria have certain properties that potentially make them more suitable for bacterial surface display applications. First, the surface proteins of Gram-positive bacteria seem to be more permissive for the insertion of extended sequences of foreign proteins that have several hundreds

\footnotetext{
* Correspondence: nguyen_hoang.xuatban@hotmail.com

${ }^{1}$ Institute of Genetics, University of Bayreuth, D-95445 Bayreuth, Germany Full list of author information is available at the end of the article
}

of amino acids, as compared with the different Gramnegative surface proteins (Samuelson et al. 2002). Second, a more obvious advantage of the Gram-positive system is that translocation through only a single membrane is required to achieve proper surface exposure of the heterologous polypeptide, while in the Gram-negative system both translocation through the cytoplasmic membrane and correct integration into the outer membrane are required for surface display. Finally, considering the practical handling of the bacteria, Gram-positive bacteria have the additional advantage of being more rigid, due to the thicker cell wall (Pagan et al. 1999; Samuelson et al. 2002), which thus allows various laboratory procedures without extensive cell lysis (Desvaux et al. 2006).

In Gram-positive bacteria, a class of surface proteins are covalently anchored on the cell wall by a transpeptidase, which has been called sortase (Srt) (Paterson and Mitchell 2004; Ton-That et al. 2004; Marraffini et al.

\section{SpringerOpen ${ }^{\circ}$}

(c) 2011 Nguyen et al; licensee Springer. This is an Open Access article distributed under the terms of the Creative Commons Attribution License (http://creativecommons.org/licenses/by/2.0), which permits unrestricted use, distribution, and reproduction in any medium, provided the original work is properly cited. 
2006; Clancy et al. 2010). Sortases are positioned at the cytoplasmic membrane via a membrane anchor located either at the $\mathrm{N}$ - or $\mathrm{C}$-terminus, contain the active site, LxTC motif (conserved residues underlined) (Marraffini et al. 2006), of which cystein is essential for the sortase activity (Ton-That et al. 1999); and recognize their substrate proteins via a common $\mathrm{C}$-terminal pentapeptide sequence, which acts as a cell wall sorting signal. Substrate proteins are not directly transferred to the cell wall, but to the peptidoglycan intermediate lipid II. So far, more than 700 putative sortase substrates encoded by more than 50 different prokaryotic genomes have been identified. The majority of these proteins are anchored by a sortase named SrtA originally identified in Staphylococcus aureus (Mazmanian et al. 1999). The number and types of proteins anchored by SrtA are predicted to vary from two in B. subtilis to up to 43 in Listeria monocytogenes (Boekhorst et al. 2005). These proteins are recognized in most cases by the pentapeptide sorting signal LPXTG (Fischetti et al. 1990).

Two putative sortase homologues of B. subtilis are YhcS and YwpE (Comfort and Clubb 2004; Pallen et al. 2001). YhcS encodes a protein of 198 amino acids carrying a transmembrane anchor at its $\mathrm{N}$-terminus and the active site motif (LxTC). YwpE encodes a small protein of 102 amino acids with the LxTC motif at the C-terminus, but it has no signal peptide at the $\mathrm{N}$-terminus (Clancy et al. 2010; Tjalsma et al. 2000). YhcS has been classified in group SrtD sortases, but there is no clear experimental evidence that class SrtD sortases recognize and anchor proteins on the surface of Gram-positive bacteria (Dramsi et al. 2005).

B. subtilis also encodes two potential sortase substrates, YfkN and YhcR, encoded by the $y f k N$ and $y h c R$ genes (Boekhorst et al. 2005; Comfort and Clubb 2004). Instead of the LPXTG motif, YfkN contains the potential sorting signal LPDTA and YhcR the sequence LPDTS. YfkN exhibits 2', 3' cyclic nucleotide phosphodiesterase and 2' (or 3') nucleotidase and 5' nucleotidase activities, a trifunctional nucleotide phosphoesterase (Chambert et al. 2003). YhcR appears to have 5'-nucleotidase activity, a property shared by LPXTG proteins from several other bacteria (Pallen et al. 2001). Its Nterminal end (residues 1 to 46) contains a signal peptide that is predicted to direct secretion by the twin-arginine translocation pathway, while the C-terminal end is a typical Gram-positive anchor (Oussenko et al. 2004). Furthermore, $y h c R$ is located adjacent to $y h c S$ on the $B$. subtilis chromosome, one of the two sortase-like proteins in $B$. subtilis. In addition, recent analysis has shown that YfkN and YhcR could accumulate in the culture medium when investigated in B. subtilis cells carrying null alleles in $y h c S$ and $y w p E$. Therefore, YfkN and YhcR could, in principle, be sorted to the cell wall by the B. subtilis sortase homologues YwpE and/or YhcS (Westers 2004).

Despite being intensively studied as a model organism and possessing two sortase-like proteins, there is no direct published evidence that $B$. subtilis might decorate its surface with sortase-dependent proteins covalently linked to the peptidoglycan. In an effort to develop $B$. subtilis as a cellular chip, we have already established a system to immobilize proteins on the surface of a $B$. subtilis strain expressing L. monocytogenes srtA (Nguyen and Schumann 2006). This work aims to analyze expression of the two putative sortases, YwpE and YhcS, and the two surface proteins, YhcR and YfkN, in order to extend tools to display proteins on the surface of any $B$. subtilis wild type strain using its own sortase(s).

\section{Materials and methods}

\section{Bacterial strains and culture conditions}

The bacterial strains and plasmids used are listed in Table 1. E. coli strain DH10B (Stratagene) was used as recipient in all cloning experiments. The B. subtilis strain 1012 was used for the construction of new strains and as a template for PCR if not mentioned otherwise. Cells were routinely grown aerobically in Luria-Bertani (LB) broth at $37^{\circ} \mathrm{C}$, and antibiotics were added as appropriate (ampicillin at $100 \mu \mathrm{g} / \mathrm{ml}$, chloramphenicol at 10 $\mu \mathrm{g} / \mathrm{ml}$, erythromycin at 1 or $100 \mu \mathrm{g} / \mathrm{ml}$, and neomycin at $10 \mu \mathrm{g} / \mathrm{ml})$.

\section{Construction of strains NDH20 and NDH21}

To measure expression of the $y h c S$ and $y w p E$ genes, transcriptional fusions between their promoter regions and the lac $Z$ reporter gene were constructed. The 5' coding region of $y h c S$ including the start codon was amplified using the primers ON59 and ON60 (Table 2), treated with EcoRI and BamHI and ligated into the integration vector pMUTIN4 (Vagner et al. 1998), cleaved with the same enzymes resulting in pNDH26. In a second experiment, the complete $y w p E$ gene including its start codon was amplified using the ON61/ON62 primer pair and inserted into pMUTIN4 yielding pNDH27. Both plasmids were transformed into B. subtilis 1012 resulting in the strains NDH20 and NDH21, respectively (Figure 1). Correct integration at the $y$ hcS locus was confirmed by PCR using ON57 and ON63 and at the $y w p E$ locus using ON55 and ON63 (Figure 1A). These PCR products were verified by sequencing using ON63. One correct transformant each was kept for further studies.

\section{Construction of strains SZ59, SZ60, NDH30, NDH31 and NDH32}

To inactivate the genes coding for the two putative sortases, their coding sequences were replaced by two 
Table 1 Bacterial strains and plasmids used

\begin{tabular}{|c|c|c|}
\hline B. subtilis & Description & Reference \\
\hline 1012 & leuA8 metB5 trpC2 hsdRM1 & (Saito et al. 1979) \\
\hline $\mathrm{NDHO3}$ & WW02 with srtA gene of Listeria monocytogenes integrated at the lacA locus & (Nguyen and Schumann 2006) \\
\hline $\mathrm{NDH} 20$ & 1012 carrying pNDH26 inserted into the chromosome & This work \\
\hline $\mathrm{NDH} 21$ & 1012 carrying pNDH27 inserted into the chromosome & This work \\
\hline $\mathrm{NDH} 30$ & 1012 yhcS :: neo $\left(\mathrm{Neo}^{\mathrm{R}}\right)$ & This work \\
\hline $\mathrm{NDH} 31$ & 1012 yhcS :: neo, ywpE :. erm $\left(\mathrm{Neo}^{\mathrm{R}}, \mathrm{Erm}^{\mathrm{R}}\right)$ & This work \\
\hline $\mathrm{NDH} 32$ & WB800 yhcs:: neo, ywpE::erm $\left(\mathrm{Neo}^{R}, \mathrm{Erm}^{\mathrm{R}}, \mathrm{Cm}^{\mathrm{R}}\right)$ & This work \\
\hline SZ59 & 1012 yhcs :: cat $\left(\mathrm{Cm}^{\mathrm{R}}\right)$ & This work \\
\hline SZ60 & 1012 ywpE :. erm $\left(\mathrm{Em}^{\mathrm{R}}\right)$ & This work \\
\hline WB800 & nprE aprE epr bpr mpr :: ble nprB :: bsr $\Delta v p r$ wprA $:$ hyg $\left(\mathrm{Cm}^{\mathrm{R}}\right)$ & (Wu et al. 2002) \\
\hline WB800N & WB800 pB-cat5-neo-cat3 $\left(\mathrm{Neo}^{\mathrm{R}}\right)$ & This work \\
\hline \multicolumn{3}{|l|}{ Plasmids } \\
\hline pBluescript II KS & & Stratagene \\
\hline PMUTIN4 & Integration vector carrying lacZ and erm & (Vagner et al. 1998) \\
\hline pNDH19 & PxylA-amyQ-fnbpB123 & (Nguyen and Schumann 2006) \\
\hline $\mathrm{pNDH} 26$ & pMUTIN4 carrying $5^{\prime}$ end of yhcs & This work \\
\hline $\mathrm{pNDH} 27$ & pMUTIN4 carrying $5^{\prime}$ end of ywpE & This work \\
\hline pNDH33 & Expression vector carrying Pgrac and $\mathrm{Cm}$ & (Phan et al. 2006) \\
\hline pNDH33-yhcs & pNDH33 carrying yhcS (Pgrac-yhcS) & This work \\
\hline pNDH33-ywpE & pNDH33 carrying ywpE (Pgrac-ywpE) & This work \\
\hline pNDH33-ywpE-yhcS & $\begin{array}{l}\text { pNDH33 carrying ywpE-yhcS } \\
\text { (Pgrac-ywpE-yhcS) }\end{array}$ & This work \\
\hline pNDH37 & Pgrac with signal sequence of amyQ & (Phan et al. 2006) \\
\hline pNDH37-amyQ & Pgrac with full length of amyQ & (Phan et al. 2006) \\
\hline pNDH88 & pHT01 with amyQ & This work \\
\hline pNDH89 & $y h c R 123$ translationally fused to amyQ & This work \\
\hline pNDH90 & yfkN123 translationally fused to amyQ & This work \\
\hline pHT01 & Expression vector carrying Pgrac and $\mathrm{Cm}$ & (Nguyen et al. 2007) \\
\hline
\end{tabular}

different antibiotic resistance markers. To obtain this goal, $y h c S$ was replaced by a chloramphenicol resistance marker resulting in strain SZ59 (yhcS::cat) and $y w p E$ by an erythromycin resistance marker (SZ60: ywpE::erm) as shown in Figure 2A and 2B. To be able to use these knockout strains with plasmids that carry a chloramphenicol resistance gene, the cat cassette in strain SZ59 was replaced by a neo cassette. First, the cat5-neo-cat 3 cassette was cloned into plasmid pBluescript II KS resulting in plasmid pB-cat5-neo-cat3. This plasmid was treated with $P v u I I$ and then transformed into strain SZ59, neomycinresistant colonies were screened for chloramphenicol sensitivity, and correct integration at the cat cassette was confirmed by PCR using ON57 and ON58 (data not shown), and one transformant was kept for further studies (NDH30). Second, chromosomal DNA of strain SZ60 was transformed into the strain NDH30; recombinants were selected on LB plates containing erythromycin and neomycin. Correct integration at the $y w p E$ locus was confirmed by PCR using ON54 and ON55, resulting in strain
NDH31. Strain NDH32 was generated by transformation of chromosomal DNA of NDH31 into WB800, followed by selection for chloramphenicol, neomycin and erythromycin resistance (strain NDH32).

\section{Construction of $B$. subtilis strain WB800N}

WB800 (Wu et al. 2002) is an eight-fold protease-deficient $B$. subtilis strain that is used for the production of secreted heterologous proteins. This strain is resistant to chloramphenicol. To be able to use it with plasmid pNDH33 derivatives all carrying a chloramphenicol resistance gene (Phan et al. 2006), a neo cassette was inserted in the middle of cat cassette resulting in strain WB800N. The PvuII-treated plasmid pB-cat5-neo-cat3 was transformed into WB800 and plated on indicator medium, calcium caseinate plates with neomycin. Colonies without halos (as compared with strain 1012) were checked for sensitivity to chloramphenicol and resistance to neomycin. One transformant was kept for further study (WB800N). 
Table 2 Oligonucleotides used

\begin{tabular}{|c|c|c|}
\hline Name & Sequence $\left(5^{\prime}\right.$ to $\left.3^{\prime}\right)$ & Description \\
\hline ON29 & GGCCATGGATCCATGATTCAAAAACGAAAGCGGACAG & $5^{\prime}$ end of amyQ \\
\hline ON42 & GGCCATGACGTCTITCTGAACATAAATGGAGACGGAC & $3^{\prime}$ end of amyQ \\
\hline ON47 & GGCCATGACGTCTTGGAAGCGACAGTTGAGTACG & 5' end of $y h c R$ \\
\hline ON48 & GAATAAGATATCTCACGTTCTGGAGGCGCTCCT & $3^{\prime}$ end of $y h c R$ \\
\hline ON49 & GGCCATGACGTCCGCATGTTTGATATTGAAGAAGC & $5^{\prime}$ end of $y f k N$ \\
\hline ON50 & AGCAGCGATATCTTATGCCTGATTCGCTCTATTCTG & $3^{\prime}$ end of $y f k N$ \\
\hline ON54 & GGCCATTTCGAAGACCTCTTIAGCTCCTTGGAAGC & $3^{\prime}$ end of erm \\
\hline ON55 & GACCTGAATGTGGAACGAGTGGAC & $5^{\prime}$ end of $y w p F$ \\
\hline ON56 & GGCCATTTCGAACCGACTGTAAAAAGTACAGTCGGCA & $3^{\prime}$ end of cat \\
\hline ON57 & CGTCTTGATCAGGATACATCTGGC & $5^{\prime}$ end of yhct \\
\hline ON58 & GAGAGCCATAAACACCAATAGCCTT & $5^{\prime}$ end of neo \\
\hline ON59 & GGCCATGAATTCAAAGGAGGAACTCCAGAACGTGAAAAAAGTTATTC & $5^{\prime}$ end of yhcs \\
\hline ON60 & CTAATACGACTCACTATAGGGAGAGGATCCCGACACCTITTCTAAATCA & $3^{\prime}$ end of yhcs \\
\hline ON61 & GGCCATGAATTCAAAGGAGGAACAACAATGCGCCGGGATCA & $5^{\prime}$ end of $y w p E$ \\
\hline ON62 & CTAATACGACTCACTATAGGGAGAGGATCCTCTTCGTGCTTCACTCTTGC & $3^{\prime}$ end of $y w p E$ \\
\hline ON63 & TCTACATCCAGAACAACCTCTGC & $5^{\prime}$ end of Pspac \\
\hline ON64 & GGCCATAGATCTATGCGCCGGGATCAAAAAATG & $5^{\prime}$ end of $y w p E$ \\
\hline ON65 & GGCCATAGATCTATGAAAAAAGTTATTCCACTATTCATCATTGC & $5^{\prime}$ end of yhcs \\
\hline ON66 & GGCCATAGATCTAGAATGAAGAAAAGCCGCAGGCACT & $3^{\prime}$ end of yhcs \\
\hline ON67 & CCAGAGATCTCAAAGGAGGAACTCCAGAACGTGAAAAAAGTTATTC & $5^{\prime}$ end of yhcs \\
\hline ON68 & AGTAAAGTTATCGGAATCGACTTAG & $5^{\prime}$ end of dnak \\
\hline ON69 & CTAATACGACTCACTATAGGGAGAAAAGTATGCAGGAACTGTGAT & $3^{\prime}$ end of dnak \\
\hline
\end{tabular}

\section{Construction of plasmids able to overexpress the two putative sortases separately and together}

To be able to overexpress $y h c S$ and/or $y w p E$ in B. subtilis under the control of the IPTG-inducible promoter $\mathrm{P}_{\text {grac }}$ (Phan et al. 2006), three different plasmids were constructed. First, the coding sequence of the $y w p E$ gene including its start codon was amplified by PCR using ON64 and ON62, the amplicon was cleaved with $B a m \mathrm{HI}$ and BglII and ligated into pNDH33 (Phan et al. 2006) at its unique $B a m \mathrm{HI}$ site resulting in pNDH33$y w p E$. The $y w p E$ gene was transcriptionally fused to $\mathrm{P}_{\text {grac }}$ and a strong ribosome-binding site (RBS) present on pNDH33. Next, gene $y h c S$ was amplified using ON65 and ON67 containing its own RBS; the PCR product was then cleaved with BglII and ligated into pNDH33$y w p E$ resulting in $\mathrm{pNDH} 33-y w p E-y h c S$. The gene $y h c S$ was also amplified using ON65 and ON66, the amplicon was treated with $B g l \mathrm{II}$ and ligated into pNDH33 at its unique $B a m \mathrm{HI}$ site resulting in pNDH33-yhcS.

\section{Construction of plasmids pNDH88, pNDH89 and pNDH90}

In order to study whether the putative B. subtilis sortases could recognize potential sorting sequences, two plasmids that allow anchoring of amyQ coding for an $\alpha$ amylase (Palva 1982) on the cell wall were constructed. In a previous report, it has been suggested that a 123amino-acids spacer between AmyQ and the sorting sequence is optimal to anchor AmyQ on the cell wall (Nguyen and Schumann 2006). Therefore, plasmids were generated, in which $a m y Q$ was translationally fused to the putative sorting sequences with the 123amino-acids spacers encoded by $y h c R$ (YhcR123) and $y f k N$ (YfkN123) under the control of the IPTG-inducible promoter Pgrac. First, the amyQ gene was generated by PCR using pKTH10 (Palva 1982) as template together with ON29 and ON42, the amplicon was treated with BamHI and AatII and ligated into pHT01 (Nguyen et al. 2007) cut with the same enzymes resulting in pNDH88. Next, the coding regions of the 3' ends of $y h c R$ and $y f k N$ including the sorting motif and the additional 123 codons, the spacer regions, were amplified using ON47/ ON48 and ON49/ON50, respectively. The amplicons were cleaved with AatII and EcoRV and inserted into pNDH88 treated with AatII and SmaI resulting in pNHD89 and pNDH90, respectively.

\section{Determination of sortase-dependent cell wall proteins}

The B. subtilis strains were inoculated to an $\mathrm{OD}_{578}$ of $0.05-0.08$ in LB medium and grown at $37^{\circ} \mathrm{C}$ in a shaking water bath. After $1 \mathrm{~h}$ of growth, $0.1 \mathrm{mM}$ IPTG was added to induce expression of $y h c S$ and/or $y w p E$ and cells corresponding to $200 \mathrm{OD}_{578}$ units were collected after about $8 \mathrm{~h}$. After sedimentation by centrifugation, the cells were resuspended in $1.5 \mathrm{ml}$ of water (final 

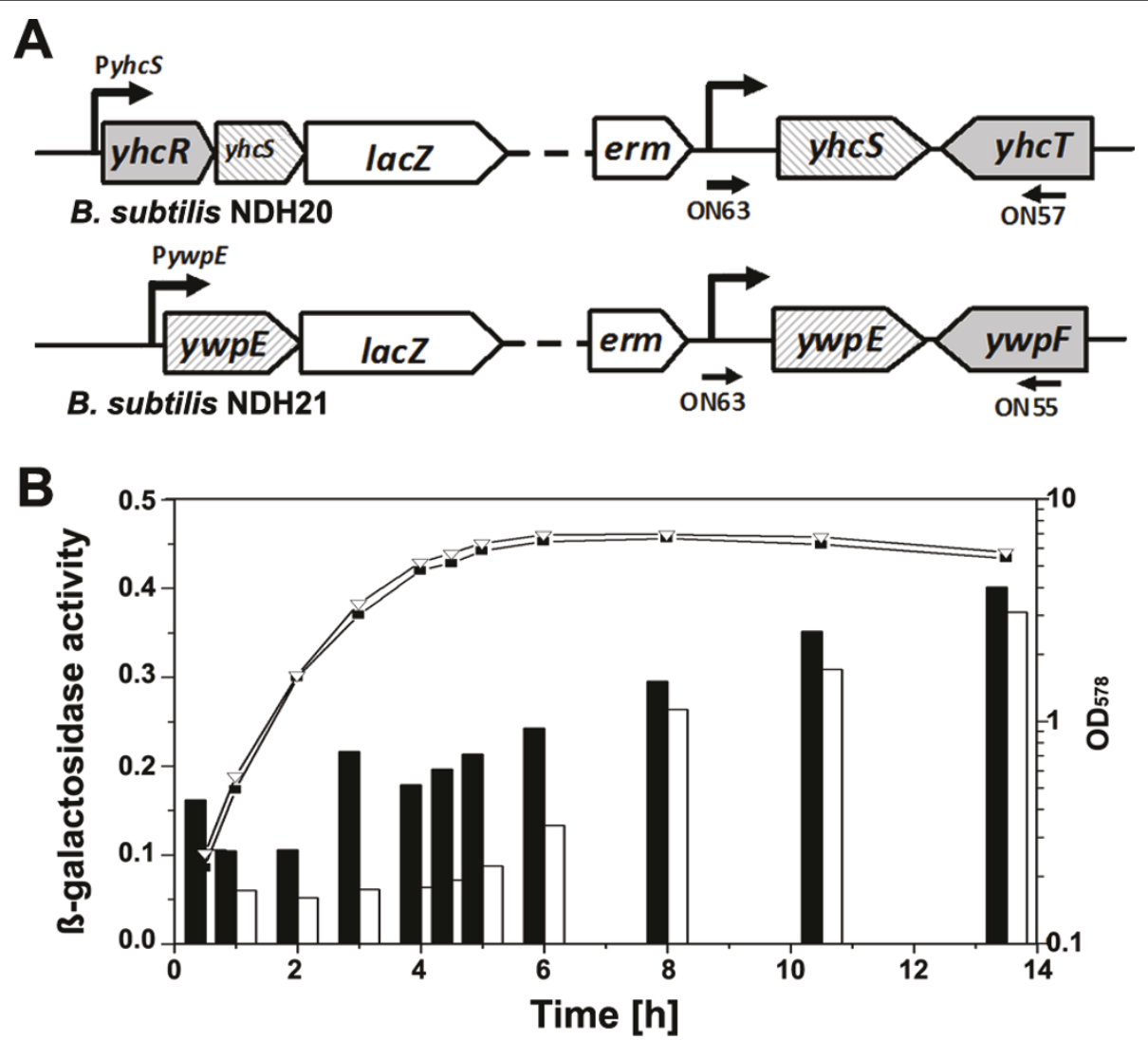

Figure 1 Transcriptional fusion of the lacZ reporter gene to the yhcS and $y w p E$ promoters. (A) Schematic representation of transcriptional fusions between the promoters of yhcS and ywpE and the lacZ reporter gene. (B) Cells containing the fusions were grown in $L B$ medium at $37^{\circ}$ $\mathrm{C}$, and aliquots were taken at the time points indicated for determination of the $\mathrm{OD}_{578}$ and for measuring the $\beta$-galactosidase activity. Strains NDH2O ('black square' and closed bars) and NDH21 ('white triangle' and open bars).

volume) containing a cocktail of protease inhibitors (Roche Diagnostics), $2 \mathrm{mM}$ EDTA and $100 \mathrm{mg} / \mathrm{ml}$ DNase I and disrupted by sonication $(12 \mathrm{~W}, 10 \times 30$ pulses with $30 \mathrm{sec}$ intervals) on ice. The unbroken cells were removed by low-speed centrifugation $(980 \times \mathrm{g})$ at $4^{\circ} \mathrm{C}$ for $10 \mathrm{~min}$. The supernatants were then centrifuged at higher speed $(21000 \times \mathrm{g})$ at $4^{\circ} \mathrm{C}$ for $15 \mathrm{~min}$ to obtain a pellet containing the envelope material. These pellets were washed three times in water containing protease inhibitors. Finally, the pellets containing peptidoglycan with cell wall proteins were resuspended in $100 \mu \mathrm{l}$ of lysozyme $(1 \mathrm{mg} / \mathrm{ml})$, incubated at $37^{\circ} \mathrm{C}$ for $45 \mathrm{~min}$ and shaken occasionally. Samples were mixed with $3 \times$ loading buffer and applied to SDS-PAGE (Figure 3). The target protein bands were extracted from the gel, and proteins were identified by MALDI-TOF mass spectrometry.

\section{Enzyme assays}

B. subtilis strains NDH20 and NDH21 (Figure 1A) containing the transcriptional fusions PyhcS-lacZ and $\mathrm{P} y w p$ E-lacZ were grown in $\mathrm{LB}$ medium at $37^{\circ} \mathrm{C}$. When an OD578 of 0.6 was reached (set as $t=0$ ) and samples were collected at the indicated time points. $\beta$-Galactosidase activity assays were performed in triplicate with soluble extracts using $\alpha$-nitrophenyl- $\beta$-D-galactoside as substrate (Miller 1972) and yielded comparable results. The activities of one representative experiment are presented. $\beta$-Galactosidase activities are given in units, where one unit is defined as $\Delta \mathrm{A}_{405} \min ^{-1} \times \mathrm{OD}_{578}{ }^{-1} \times$ $10^{-3}$, in which $\mathrm{OD}_{578}$ is the optical density of the growth culture.

To measure $\alpha$-amylase activity, the $B$. subtilis strains 1012/pNDH37, 1012/pNDH37-amyQ, NDH30/pNDH89, NDH03/pNDH19 and 1012/pNDH89, three different clones for each strain were grown in LB medium containing chloramphenicol $(10 \mu \mathrm{g} / \mathrm{ml})$ at $37^{\circ} \mathrm{C}$. When the $\mathrm{OD}_{578}$ of the cultures reached to mid-log phase $\left(\mathrm{OD}_{578}\right.$ $0.6), 0.5 \mathrm{mM}$ IPTG and $0.5 \%$ xylose were added to all cultures to induce production of sortase $\mathrm{A}$ in the NDH03 strains, amylase (AmyQ, from pNDH37-amyQ) and the hybrids AmyQ-FnbpB123 (from pNDH19) and AmyQ-YhcR123 (pNDH89). Cells were separated from the growth medium by centrifugation, washed twice 



B. subtilis 1012
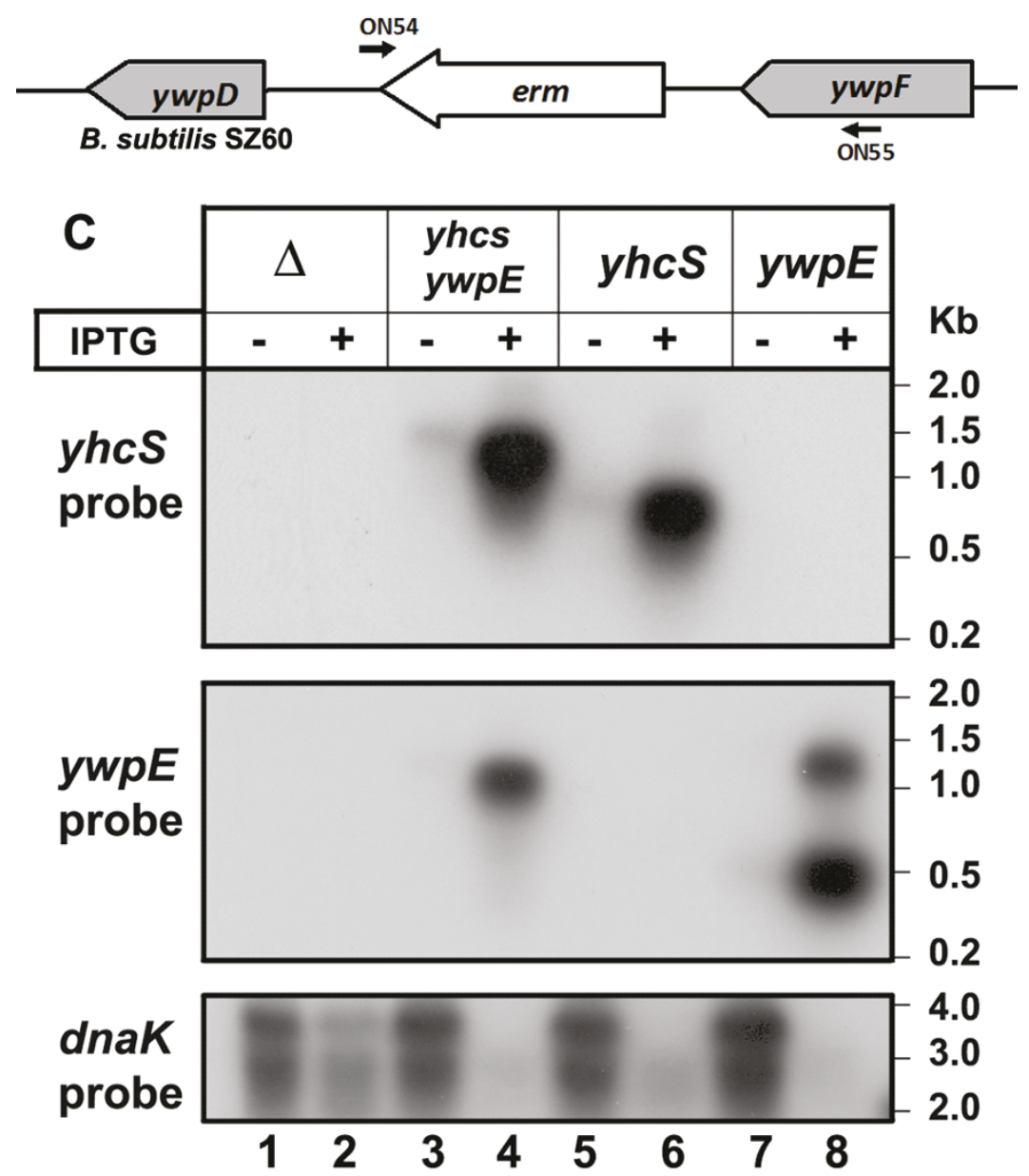

Figure 2 Expression of yhcS and/or ywpE in strain NDH31 ( $\Delta y h c S$ and $\Delta y w p E$ ) from plasmids by Northern blot. (A, B) Schematic representation of chromosomal regions of the knockout strains SZ59, SZ60 and NDH30. The positions of ONs used for verification of the null alleles by PCR are indicated. Three pairs of primers have been used: ON54 and ON55 specifically recognize chromosomal DNA of strain SZ60 (1617-bp PCR product), ON56 and ON57 strain SZ59 (1486-bp PCR product) and ON57 and ON58 strain NDH30 (1602-bp PCR product). (C) Expression of yhcS and/or ywpE in strain NDH31 with different plasmids (pNDH33, pNDH33-yhcS, pNDH33-ywpE and pNDH33-ywpE-yhcS. Either a total of $0.25 \mu \mathrm{g}$ (lanes 4,6 and 8) or $5 \mu \mathrm{g}$ of RNA (lanes 1, 2, 3, 5, 7) were loaded per lane. RNA markers are indicated on the right margin. 


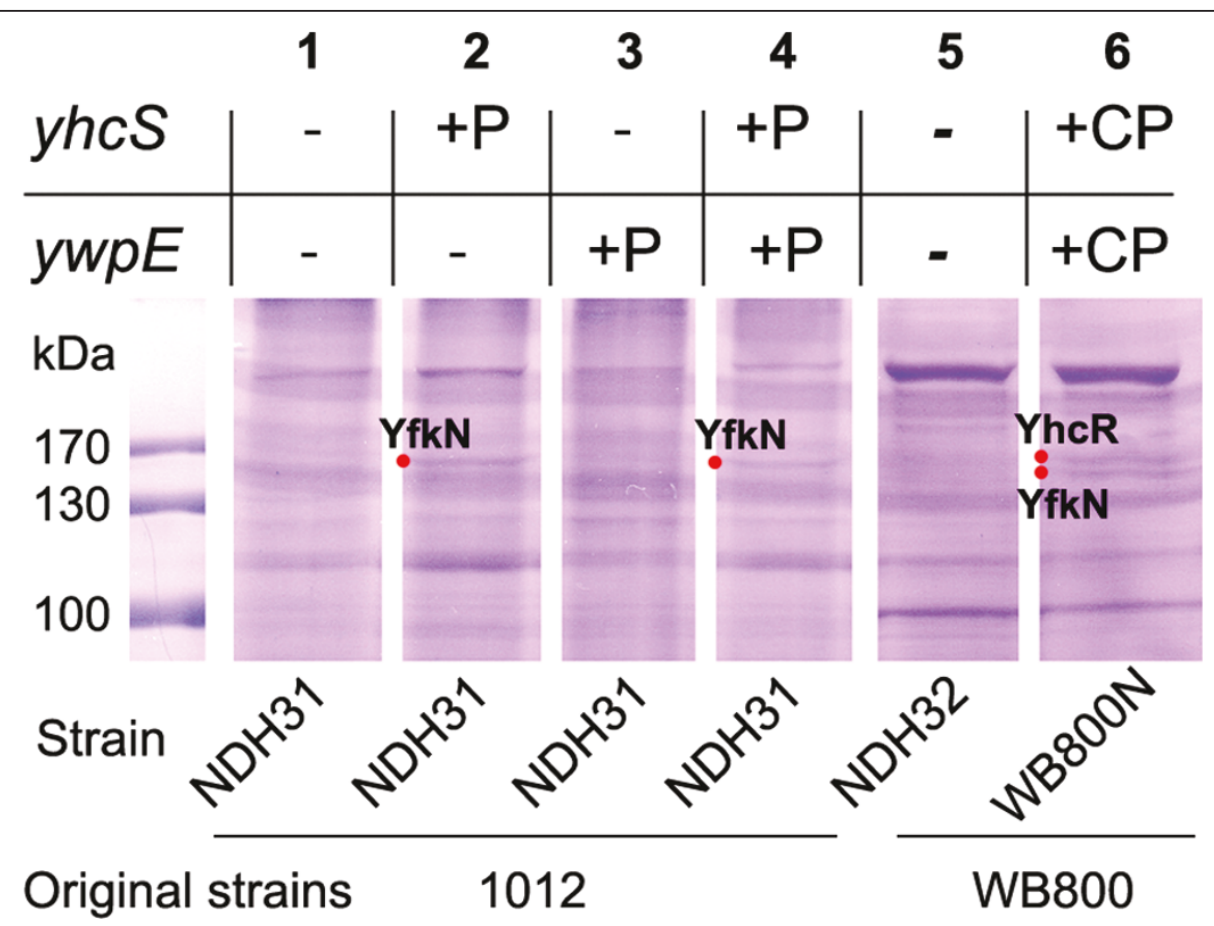

Figure 3 Protein patterns of the putative sortase knockout strains. Samples were collected $8 \mathrm{~h}$ after induction. The cells were sonicated, followed by intensive washing and lysozyme treatment. Samples for SDS-PAGE and Coomassie blue staining were prepared as described in Materials and methods. The following strains have been analyzed: 1, NDH31/pNDH33 ( $\Delta y$ hcS $\triangle y w p E) ; 2, N D H 31 / p N D H 33-y h c S(\Delta y w p E)(+P)$; 3 , NDH31/pNDH33-ywpE; 4, NDH31/pNDH33-ywpE-yhcS; 5, NDH32 ( $\triangle$ yhcS $\triangle y w p E$ ) derived from WB800 and 6, WB800N/pNDH33-ywpE-yhcS carrying yhcS and ywpE both on the chromosome and on the plasmid (+CP) were investigated. Strain NDH31 was derived from B. subtilis 1012, and NDH32 and WB800N were derived from B. subtilis WB800. The size of molecular weight standards is indicated on the left margin.

with the medium and once with PBS buffer ( $\mathrm{pH} 7.4)$ and finally resuspended in PBS buffer. Cells corresponding to $\mathrm{OD}_{578}$ of 0.2 in $100 \mu \mathrm{l}$ were used to measure the $\alpha$-amylase activities. As a control, the enzymatic activity secreted in the supernatant from the strain 1012/ pNDH37-amyQ was also determined and was set at $100 \%$.

\section{RNA extraction and Northern blot analysis}

B. subtilis cells were grown and induced as described under enzyme assays. Strains containing plasmids pNDH33-yhcS and pNDH33-ywpE-yhcS were induced by $0.1 \mathrm{mM}$ IPTG, and the cells were killed by addition of "killing buffer" ( $5 \mathrm{mM} \mathrm{MgCl}_{2}, 20 \mathrm{mM} \mathrm{NaN}_{3}, 20 \mathrm{mM}$ Tris-HCl; pH7.5). Total RNA was extracted using the protocol for isolation of RNA from yeast with modification (Robert 1998). The cell walls were digested by addition of lysozyme $(1 \mathrm{mg} / \mathrm{ml})$ on ice and the samples were then heated at $95^{\circ} \mathrm{C}$ for 5 min before addition of phenol. The RNA concentration was measured at $280 \mathrm{~nm}$ and $10 \mu \mathrm{g}$ of total RNA was loaded in each well. Northernblot analyses were performed as described (Roche Company 2003) with antisense RNAs produced against the putative sortase mRNAs. Hybridizations specific for the putative sortase genes were carried out with digoxigenin (DIG)-labelled riboprobe RNAs synthesized in vitro with T7 RNA polymerase from PCR products equipped with a promoter recognized by that polymerase (DIG RNA labelling kit; Roche Diagnostics, Mannheim, Germany). Pairs of primers ON59/ON60 and ON61/ON62 were used to amplify an internal part of the $y h c S$ and the complete $y w p E$ gene, respectively. The ON68/ON69 primers were used to amplify dnaK used as a loading control (Homuth et al. 1999).

\section{Results}

Natural expression of yhcS and ywpE

B. subtilis $y$ hcS codes for a putative sortase of 198 amino acids with one predicted transmembrane domain, while $y w p E$ encodes a predicted cytoplasmic protein of only 102 amino acid residues (Comfort and Clubb 2004; Pallen et al. 2001). The latter exhibits $23 \%$ sequence identity with the C-terminal domain of SrtA. To follow expression of the two genes during growth, each promoter was fused to the lacZ reporter gene (Figure $1 \mathrm{~A}$ ), and the $\beta$-galactosidase activity was measured during growth. First, expression of the $y w p E$ gene turned out to be lower than that of the $y h c S$ gene during exponential and 
early stationary phase, but both activities were comparable during late stationary phase (Figure 1B). Second, expression of both genes increased over time to be highest during late stationary phase. We conclude from this result that both putative sortase genes are preferentially expressed after cells have entered the stationary phase.

Next, we analyzed transcription of the two genes in the B. subtilis 1012 wild type strain directly by Northern blot to confirm these results. Total RNA was isolated at different time points during growth and hybridized against gene-specific DIG-labelled antisense RNA. A transcript of about $4.5 \mathrm{~kb}$ could be detected after $6 \mathrm{~h}$ of growth with a further increase at $8 \mathrm{~h}$ (Figure 4 ). The 4.5 -kb transcript corresponds by size to the bicistronic $y h c R-y h c S$ operon (Price et al. 2005). The smaller bands below the $4.5-\mathrm{kb}$ transcript most probably represent processing or/and degradation products. The failure to detect a $y w p E$-specific transcript even when using a large amount of RNA (30 $\mu \mathrm{g})$ could indicate instability (data not shown). When both genes were expressed artificially from an IPTG-inducible promoter, their bicistronic transcript was produced in high amounts (Figure 4, left lane) indicating full stability under these conditions. In conclusion, putative sortase $y h c S$ gene is expressed preferentially at the late stationary phase while $y p w E$ expression could not be detected in the Northern blot analysis. This might point to a role of at least one of these two enzymes (YhcS) in anchoring proteins during stationary phase to the cell wall.

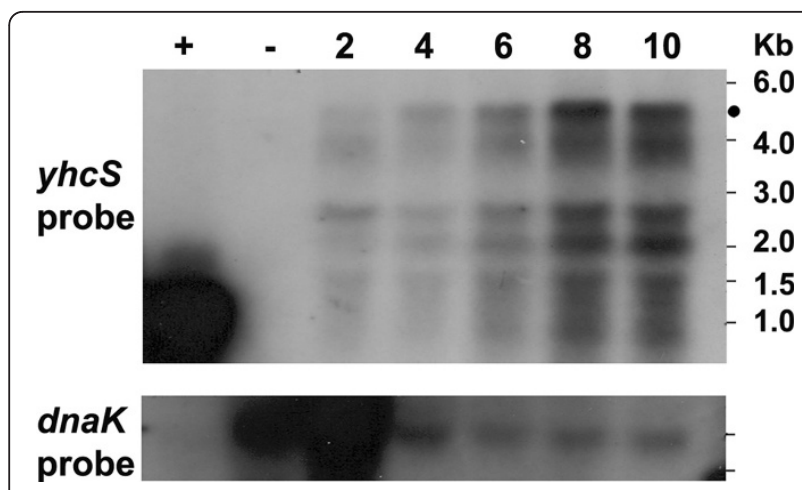

Figure 4 Detection of the expression of yhcS by Northern blot. Three different $B$. subtilis strains were grown in LB medium and aliquots were analysed by Northern blot using either yhcS (upper panel), ywpE (middle panel) or dnaK antisense RNA (lower panel, loading control). +, strain NDH31/pNDH33-ywpE-yhcS where both genes coding for putative sortases were artificially expressed from an IPTG-dependent promoter; -, strain NDH31 where both putative sortase genes have been deleted. Lanes 2 to 10, aliquots from wild type strain 1012 were withdrawn at 2, 4, 6, 8 and $10 \mathrm{~h}$ after inoculation.
Search for yhcS and/or ywpE-dependent surface proteins To identify putative sortase-dependent substrate proteins, the three knockout strains SZ59 ( $\Delta y h c S)$, SZ60 $(\Delta y w p E)$ and NDH31 ( $\triangle y h c S$ and $\Delta y w p E)$ were constructed (Figure 2A and $2 \mathrm{~B}$ ). All three mutant strains together with the isogenic wild-type strain were incubated in LB medium for $8 \mathrm{~h}$ corresponding to the late stationary phase and analyzed for the presence of cell wall anchored proteins as described under Materials and methods. No difference in the protein pattern could be detected (data not shown). We conclude that the amount of proteins anchored is not sufficient to be detected either due to the low amount of sortase enzymes or due to these two enzymes, or due to a mixture of both. Therefore, we decided to repeat this experiment with strains, where either $y w p E$ or $y h c S$ or both genes could be expressed together using the IPTGinducible promoter, Pgrac from plasmid pNDH33. Expression of these genes was analyzed by Northern blot. While induced expression of $y h c S$ and $y w p E$ yielded the expected RNAs of about 1 and $0.5 \mathrm{~kb}$, respectively, the artificial bicistronic operon led to the detection of an RNA larger than $1 \mathrm{~kb}$ (Figure 2C). These experiments clearly demonstrate that both genes can be expressed if fused to a strong promoter.

Interestingly, strains that restored expression of $y w p E$ and/or yhcS exhibited two YhcS-dependent proteins with molecular weights between $130 \mathrm{kDa}$ and $170 \mathrm{kDa}$ which appeared in the strains that express $y h c S$ (Figure 3, lanes 2 and 4). In addition, when the strain WB800N, deficient for eight different proteases (Wu et al. 2002), carrying plasmid pNDH33-ywpE-yhcS was analyzed several bands became visible. Among them a band running with a molecular mass of $140 \mathrm{kDa}$ seems to be a doublet (Figure 3, lane 6). These protein bands were then extracted and proteins were determined by MALDITOF mass spectrometry. As we expected one of these proteins is YhcR and the other is $\mathrm{YfkN}$, both containing the secretional sequence and potential sorting signal.

\section{Displaying AmyQ on the surface using the sorting sequences}

Next, we asked whether the two putative sortases YhcS and YwpE could anchor the proteins YhcR and YfkN on the surface of B. subtilis using their sorting signal. We fused the $a m y Q$-encoded $\alpha$-amylase to the potential sorting signals of the two proteins together with an 123amino-acid spacer region resulting in pNDH89 (AmyQYhcR123) and pNDH90 (AmyQ-YfkN123). These plasmids were transformed into strains SZ60 ( $\Delta y w p E)$, NDH30 $(\Delta y h c S)$, NDH31 $(\Delta y h c, \Delta y w p E)$ and 1012. To determine the amylase activity on the cell surface, strain NDH03/pNDH19 that has been described to immobilize amylase on the surface (Nguyen and Schumann 2006) 
and strain 1012/pNDH37-amyQ (Phan et al. 2006) that secretes the amylase into the culture medium were used as positive and negative control, respectively. Cells of these strains were grown as described under Materials and methods for Western-blot (Figure 5), and samples were collected at the appropriate time points for measuring the amylase activities (Figure 6).

When the $\alpha$-amylase carrying the YfkN123 sorting sequence was tested, anchored protein was hardly detected in the strain expressing either $y h c S$ or $y h c S$ and $y w p E 8 \mathrm{~h}$ after induction (data not shown). When the YhcR123 motif was tested, a strong anchoring occurred in the presence of $\mathrm{YhcS}$ with some further increase upon additional synthesis of YwpE (Figure 5, $8 \mathrm{~h}$ and 12 h). But since a substantial amount of AmyQ-YhcR123 is already present in the absence of both putative sortases, these hybrid protein molecules might be retained in the cytoplasmic membrane due to the presence of a

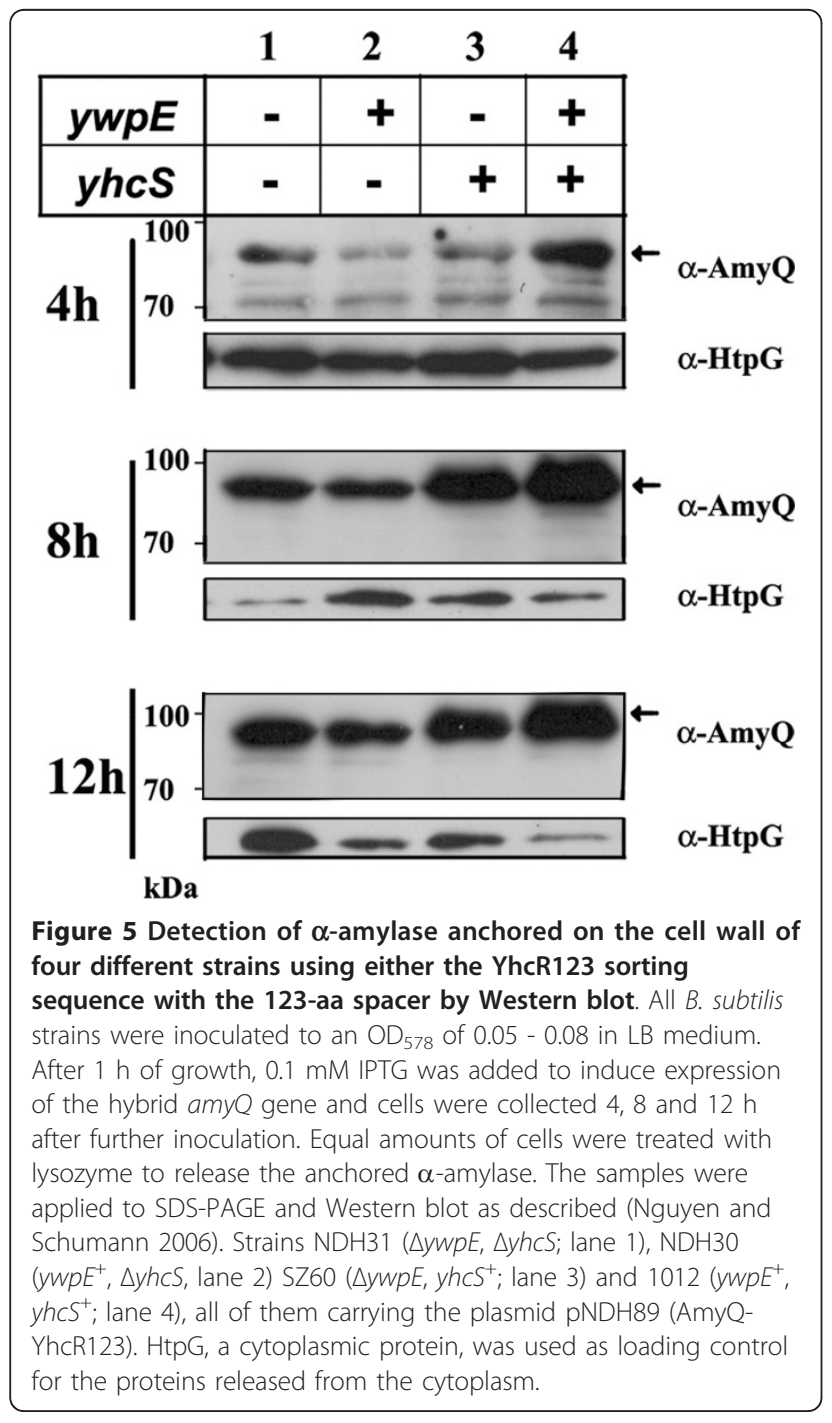

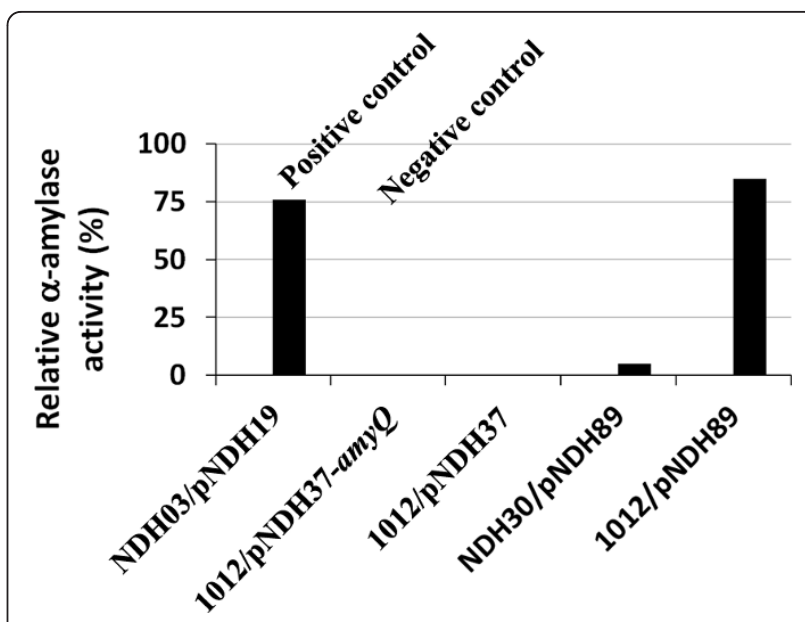

Figure $6 \alpha$-Amylase activities in the presence and absence of potential sortases. The following strains were analyzed: 1012/ pNDH37 (basic expression vector with the IPTG-inducible promoter Pgrac and the signal sequence of amyQ), 1012/pNDH37-amyQ (secretes $\alpha$-amylase into the medium), NDH03/pNDH19 (contains the xylose-inducible srtA of $L$. monocytogenes and amyQ fused to the sorting sequence of FnBPB), NDH30 ( $\triangle y$ hcS)/pNDH89 (AmyQYhcR123) and 1012/pNDH89. Cells were grown to the mid logphase and then, $0.5 \mathrm{mM}$ IPTG and 0.5\% xylose were added into all five cultures to induce production of sortase A (strain NDH03), wildtype amylase (pNDH37-amyQ) and hybrid $\alpha$-amylase (from pNDH19 and pNDH89). Samples were collected after $4 \mathrm{~h}$ of induction and the cells were separated from the growth medium by centrifugation. $\alpha$-Amylase activities were determined with whole cells that the number of cells are identical in all probes and with the supernatant from strain 1012/pNDH37-amyQ. The activities were presented as relative activity (\%), where the activity measured with the supernatant from 1012/pNDH37-amyQ was set at 100\%.

hydrophobic region being part of the sorting sequence. The presence of $\alpha$-amylase attached to cells in the absence of potential sortases could also be observed with whole cells $4 \mathrm{~h}$ after induction (Figure 6 NDH30/ pNDH89, 5\% activity) when compared with the negative controls (0\% activity for both 1012/pNDH37 and 1012/ pNDH37-amyQ), and the positive control (76\% activity for NDH03/pNDH19). Additionally, $\alpha$-amylase activity of the sample that produces both potential sortases and the hybrid protein, AmyQ-YhcR123 (Figure 6, 1012/ pNDH89) was as high as the positive control (Figure 6, NDH03/pNDH19); and the same results could be measured for samples collected after $2 \mathrm{~h}$ and $8 \mathrm{~h}$ of induction (data not shown). This activity-based measurement confirmed that the fusion YhcR123-AmyQ could be displayed on the surface of B. subtilis. In summary, these results strongly suggest that the $y h c S$ gene codes for a true sortase able to anchor at least YhcR on the cell wall of B. subtilis cell and we suggest renaming it to $s r t D$. This work could also propose an alternative way to immobilize a heterologous protein on the cell wall of $B$. subtilis using a fusion form of YhcR sorting sequence. 


\section{Discussion}

Using bioinformatics tools, two sortase-like genes and two substrate proteins have been identified (Comfort and Clubb 2004; Pallen et al. 2001; Boekhorst et al. 2005). We could show here that the putative sortase genes $y w p E$ and $y h c S$ are preferentially expressed in the late stationary phase. This finding suggests that these enzymes fulfill their task mainly during that growth phase. Furthermore, we could demonstrate that the two putative sortase-dependent substrate proteins, YfkN and YhcR, can be anchored on the cell wall in the presence of YhcS. In terms of application, this work demonstrated that the YhcR sorting sequence can be specifically used to display heterologous proteins on the cell-wall of $B$. subtilis cells. The B. subtilis cell wall contains peptide crosslinks identical to those present in the L. monocytogenes cell walls. This suggests that the crosslink of potential surface proteins to the peptidoglycan is formed by the nucleophilic attack of the amino group of m-diaminopimelic acid cross-bridge within the lipid II precursor as in the case of L. monocytogenes (Dhar et al. 2000).

Sortases have been used to anchor heterologous proteins on the cell wall of different Gram-positive bacterial species (Wernerus and Stahl 2004; Tsukiji and Nagamune 2009; Clancy et al. 2010). In a previous study, we established a system to display recombinant proteins on the cell wall of B. subtilis (Nguyen and Schumann 2006). It consists of the $L$. monocytogenes $\operatorname{srt} A$ gene fused to an inducible promoter and inserted into the chromosome and a plasmid-based expression system with the $S$. aureus FnBPB sorting signal. Since the AmyQ-FnBPB123 fusion protein could be hardly detected in the absence of the L. monocytogenes sortase, it implies that the YhcS sortase could not recognize the sorting signal present in this protein (LPxTG). Here, we show that the YhcS sortase could immobilize YhcR and YfkN with their putative sorting signals LPDTS and LPDTA, respectively. This motif is close to the one recognized by SrtD of B. anthracis (LPNTA) (Maresso and Schneewind 2008) and indicates that the YhcS protein really belongs to the group SrtD sortases. Therefore, we suggest renaming the gene $y h c S$ into $s r t D$.

We are interested in using engineered bacteria as delivery vectors for biopharmaceutical purposes. B. subtilis would be an ideal organism since (i) it is a generally recognized as safe (GRAS) organism, (ii) can localize in tumours ( $\mathrm{Yu}$ et al. 2008) enabling to use engineered $B$. subtilis cells for cancer therapy, and (iii) has a large body of information available to control protein expression in the cytoplasm, on the cell surface and secreted into the culture medium (Pohl and Harwood 2010; Schumann 2007). Different protein expression systems have been developed using small inducer molecules such as xylose (Kim et al. 1996), IPTG (Phan et al. 2010; Nguyen et al. 2005), arabinose (De Lencastre and de SaNogueira 2000), tetracycline (Kamionka et al. 2005), glycine (Phan and Schumann 2007) and lysine (Phan and Schumann 2009). (iiii) Additionally, surface displaying systems are available to immobilize proteins (Nguyen and Schumann 2006) that can bind to the surface of mammalian cells facilitating the internalization of the engineered bacteria (Bierne et al. 2002). Engineered bacteria expressing an appropriate surface protein facilitating their internalization into mammalian cells, furthermore a protein enhancing their survival in the host cells and a functional protein are called cellular chips or microbiorobots. Microbiorobots can be used as a vaccine delivery vector (Paccez et al. 2007) or for the development of a cancer therapy in the near future.

\section{Acknowledgements}

We thank Dr. Stephan Zellmeier for construction of the strains SZ59 and SZ60 and Dr. Haike Antelmann for her help with proteomics. We also thank the DLR (VNB02/B03) and the MOST (Life Science-643204) for partial financial support.

\section{Author details}

${ }^{1}$ Institute of Genetics, University of Bayreuth, D-95445 Bayreuth, Germany ${ }^{2}$ Center for Bioscience and Biotechnology, University of Science, Vietnam National University, 227 Nguyen Van Cu, District 5, Ho Chi Minh City, Vietnam ${ }^{3}$ Laboratory of Molecular Biotechnology, University of Science, Vietnam National University, 227 Nguyen Van Cu, District 5, Ho Chi Minh City, Vietnam

\section{Competing interests}

The authors declare that they have no competing interests.

Received: 8 July 2011 Accepted: 21 July 2011 Published: 21 July 2011

\section{References}

Bierne H, Mazmanian SK, Trost M, Pucciarelli MG, Liu G, Dehoux P, Jansch L, Garcia-del PF, Schneewind O, Cossart P (2002) Inactivation of the srtA gene in Listeria monocytogenes inhibits anchoring of surface proteins and affects virulence. Mol Microbiol 43(4):869-881. doi:10.1046/j.1365-2958.2002.02798.x.

Boekhorst J, de Been MW, Kleerebezem M, Siezen RJ (2005) Genome-wide detection and analysis of cell wall-bound proteins with LPXTG-like sorting motifs. J Bacteriol 187(14):4928-4934. doi:10.1128/JB.187.14.4928-4934.2005.

Chambert R, Pereira Y, Petit-Glatron MF (2003) Purification and characterization of YfkN, a trifunctional nucleotide phosphoesterase secreted by Bacillus subtilis. J Biochem(Tokyo) 134(5):655-660.

Clancy KW, Melvin JA, MCCafferty DG (2010) Sortase transpeptidases: insights into mechanism, substrate specificity, and inhibition. Biopolymers 94(4):385-396. doi:10.1002/bip.21472

Comfort D, Clubb RT (2004) A comparative genome analysis identifies distinct sorting pathways in gram-positive bacteria. Infect Immun 72(5):2710-2722. doi:10.1128/IAl.72.5.2710-2722.2004.

Daugherty PS (2007) Protein engineering with bacterial display. Curr Opin Struct Biol 17(4):474-480. doi:10.1016/j.sbi.2007.07.004.

De Lencastre H, de Sa-Nogueira I (2000) Highly regulatable promoter for heterologous gene expression. US6030807(US6030807)

Desvaux M, Dumas E, Chafsey I, Hebraud M (2006) Protein cell surface display in Gram-positive bacteria: from single protein to macromolecular protein structure. FEMS Microbiol Lett 256(1):1-15. doi:10.1111/j.15746968.2006.00122.x

Dhar G, Faull KF, Schneewind O (2000) Anchor structure of cell wall surface proteins in Listeria monocytogenes. Biochemistry 39(13):3725-3733. doi:10.1021/bi992347o. 
Dramsi S, Trieu-Cuot P, Bierne H (2005) Sorting sortases: a nomenclature proposal for the various sortases of Gram-positive bacteria. Res Microbiol 156(3):289-297. doi:10.1016/j.resmic.2004.10.011.

Fischetti VA, Pancholi V, Schneewind O (1990) Conservation of a hexapeptide sequence in the anchor region of surface proteins from gram-positive cocci. Mol Microbiol 4(9):1603-1605. doi:10.1111/j.1365-2958.1990.tb02072.x.

Homuth G, Mogk A, Schumann W (1999) Post-transcriptional regulation of the Bacillus subtilis dnaK operon. Mol Microbiol 32(6):1183-1197. doi:10.1046/ j.1365-2958.1999.01428.x.

Kamionka A, Bertram R, Hillen W (2005) Tetracycline-dependent conditional gene knockout in Bacillus subtilis. Appl Environ Microbiol 71(2):728-733. doi:10.1128/AEM.71.2.728-733.2005.

Kim L, Mogk A, Schumann W (1996) A xylose-inducible Bacillus subtilis integration vector and its application. Gene 181(1-2):71-76. doi:10.1016/ S0378-1119(96)00466-0.

Maresso AW, Schneewind O (2008) Sortase as a target of anti-infective therapy. Pharmacol Rev 60(1):128-141. doi:10.1124/pr.107.07110.

Marraffini LA, Dedent AC, Schneewind O (2006) Sortases and the art of anchoring proteins to the envelopes of gram-positive bacteria. Microbiol Mol Biol Rev 70(1):192-221. doi:10.1128/MMBR.70.1.192-221.2006.

Mazmanian SK, Liu G, Ton-That H, Schneewind O (1999) Staphylococcus aureus sortase, an enzyme that anchors surface proteins to the cell wall. Science 285(5428):760-763. doi:10.1126/science.285.5428.760.

Miller JH (1972) Experiments in molecular genetics. Cold Spring Harbor Laboratory, New York

Nguyen HD, Nguyen QA, Ferreira RC, Ferreira LC, Tran LT, Schumann W (2005) Construction of plasmid-based expression vectors for Bacillus subtilis exhibiting full structural stability. Plasmid 54(3):241-248. doi:10.1016/j. plasmid.2005.05.001.

Nguyen HD, Phan TTP, Schumann W (2007) Expression vectors for the rapid purification of recombinant proteins in Bacillus subtilis. Current Microbiology 55(2):89-93. doi:10.1007/s00284-006-0419-5.

Nguyen HD, Schumann W (2006) Establishment of an experimental system allowing immobilization of proteins on the surface of Bacillus subtilis cells. J Biotechnol 122:473-482. doi:10.1016/j.jbiotec.2005.09.012.

Oussenko IA, Sanchez R, Bechhofer DH (2004) Bacillus subtilis YhcR, a highmolecular-weight, nonspecific endonuclease with a unique domain structure. J Bacteriol 186(16):5376-5383. doi:10.1128/JB.186.16.5376-5383.2004.

Paccez JD, Nguyen HD, Luiz WB, Ferreira RC, Sbrogio-Almeida ME, Schumann W, Ferreira LC (2007) Evaluation of different promoter sequences and antigen sorting signals on the immunogenicity of Bacillus subtilis vaccine vehicles. Vaccine 25(24):4671-4680. doi:10.1016/j.vaccine.2007.04.021.

Pagan R, Manas P, Raso J, Condon S (1999) Bacterial resistance to ultrasonic waves under pressure at nonlethal (manosonication) and lethal (manothermosonication) temperatures. Appl Environ Microbiol 65(1):297-300

Pallen MJ, Lam AC, Antonio M, Dunbar K (2001) An embarrassment of sortases a richness of substrates? Trends Microbiol 9(3):97-102. doi:10.1016/50966842X(01)01956-4.

Palva I (1982) Molecular cloning of alpha-amylase gene from Bacillus amyloliquefaciens and its expression in B. subtilis. Gene 19(1):81-87. doi:10.1016/0378-1119(82)90191-3.

Paterson GK, Mitchell TJ (2004) The biology of Gram-positive sortase enzymes. Trends Microbiol 12(2):89-95. doi:10.1016/j.tim.2003.12.007.

Phan TT, Nguyen HD, Schumann W (2010) Establishment of a simple and rapid method to screen for strong promoters in Bacillus subtilis. Protein Expr Purif $71(2): 174-178$. doi:10.1016/j.pep.2009.11.010.

Phan TT, Schumann W (2007) Development of a glycine-inducible expression system for Bacillus subtilis. J Biotechnol 128(3):486-499. doi:10.1016/j. jbiotec.2006.12.007.

Phan TT, Schumann W (2009) Transcriptional analysis of the lysine-responsive and riboswitch-regulated lysC gene of Bacillus subtilis. Curr Microbiol 59(4):463-468. doi:10.1007/s00284-009-9461-4.

Phan TTP, Nguyen HD, Schumann W (2006) Novel plasmid-based expression vectors for intra- and extracellular production of recombinant proteins in Bacillus subtilis. Protein Expr Purif 46:189-195. doi:10.1016/j.pep.2005.07.005.

Pohl S, Harwood CR (2010) Heterologous protein secretion by Bacillus species: From the cradle to the grave. Adv Appl Microbiol 73:1-25

Price MN, Huang KH, Alm EJ, Arkin AP (2005) A novel method for accurate operon predictions in all sequenced prokaryotes. Nucleic Acids Res 33(3):880-892. doi:10.1093/nar/gki232.
Robert EFJ (1998) Isolation of RNA from yeast. In: Farrell EFJ (ed) RNA isolation strategies: A laboratory guide for isolation and characterization, 2nd edn. Academic Press, San Diego pp 92-94

Roche company (2003) DIG Application Manual for Filter Hybridization, 1-209

Saito H, Shibata T, Ando T (1979) Mapping of genes determining nonpermissiveness and host-specific restriction to bacteriophages in Bacillus subtilis Marburg. Mol Gen Genet 170(2):117-122. doi:10.1007/BF00337785.

Samuelson P, Gunneriusson E, Nygren PA, Stahl S (2002) Display of proteins on bacteria. J Biotechnol 96(2):129-154. doi:10.1016/S0168-1656(02)00043-3.

Schumann W (2007) Production of recombinant proteins in Bacillus subtilis. Adv Appl Microbiol 62:137-189

Tjalsma H, Bolhuis A, Jongbloed JD, Bron S, van Dijl JM (2000) Signal peptidedependent protein transport in Bacillus subtilis: a genome-based survey of the secretome. Microbiol Mol Biol Rev 64(3):515-547. doi:10.1128/ MMBR.64.3.515-547.2000.

Ton-That H, Liu G, Mazmanian SK, Faull KF, Schneewind O (1999) Purification and characterization of sortase, the transpeptidase that cleaves surface proteins of Staphylococcus aureus at the LPXTG motif. Proc Natl Acad Sci USA 96(22):12424-12429. doi:10.1073/pnas.96.22.12424.

Ton-That H, Marraffini LA, Schneewind O (2004) Protein sorting to the cell wall envelope of Gram-positive bacteria. Biochim Biophys Acta 1694(1-3):269-278. doi:10.1016/.bbamcr.2004.04.014.

Tsukiji S, Nagamune T (2009) Sortase-mediated ligation: a gift from Gram-positive bacteria to protein engineering. Chembiochem 10(5):787-798. doi:10.1002/ cbic.200800724.

Vagner V, Dervyn E, Ehrlich SD (1998) A vector for systematic gene inactivation in Bacillus subtilis. Microbiology 144:3097-3104. doi:10.1099/00221287-144-113097.

Wernerus H, Stahl S (2004) Biotechnological applications for surface-engineered bacteria. Biotechnol Appl Biochem 40(Pt 3):209-228

Westers H (2004) Genome engineering and protein secretion stress in the BACELL factory. Dissertation. University of Groningen, The Netherlands

Wu SC, Yeung JC, Duan Y, Ye R, Szarka SJ, Habibi HR, Wong SL (2002) Functional production and characterization of a fibrin-specific single-chain antibody fragment from Bacillus subtilis: effects of molecular chaperones and a wallbound protease on antibody fragment production. Appl Environ Microbiol 68(7):3261-3269. doi:10.1128/AEM.68.7.3261-3269.2002.

Yu YA, Zhang Q, Szalay AA (2008) Establishment and characterization of conditions required for tumor colonization by intravenously delivered bacteria. Biotechnol Bioeng 100(3):567-578. doi:10.1002/bit.21785.

doi:10.1186/2191-0855-1-22

Cite this article as: Nguyen et al:: Analysis and application of Bacillus subtilis sortases to anchor recombinant proteins on the cell wall. AMB Express 2011 1:22.

\section{Submit your manuscript to a SpringerOpen ${ }^{\circ}$ journal and benefit from:}

- Convenient online submission

- Rigorous peer review

- Immediate publication on acceptance

- Open access: articles freely available online

- High visibility within the field

- Retaining the copyright to your article

Submit your next manuscript at $\boldsymbol{\nabla}$ springeropen.com 